Bull. Soc. math. France

130 (3), 2002, p. 337-347

\title{
ON THE SIZE OF THE SETS OF GRADIENTS OF BUMP FUNCTIONS AND STARLIKE BODIES ON THE HILBERT SPACE
}

\author{
By Daniel Azagra \& Mar Jiménez-Sevilla
}

\begin{abstract}
We study the size of the sets of gradients of bump functions on the Hilbert space $\ell_{2}$, and the related question as to how small the set of tangent hyperplanes to a smooth bounded starlike body in $\ell_{2}$ can be. We find that those sets can be quite small. On the one hand, the usual norm of the Hilbert space $\ell_{2}$ can be uniformly approximated by $C^{1}$ smooth Lipschitz functions $\psi$ so that the cones generated by the ranges of its derivatives $\psi^{\prime}\left(\ell_{2}\right)$ have empty interior. This implies that there are $C^{1}$ smooth Lipschitz bumps in $\ell_{2}$ so that the cones generated by their sets of gradients have empty interior. On the other hand, we construct $C^{1}$-smooth bounded starlike bodies $A \subset \ell_{2}$, which approximate the unit ball, so that the cones generated by the hyperplanes which are tangent to $A$ have empty interior as well. We also explain why this is the best answer to the above questions that one can expect.
\end{abstract}

Texte reçu le 22 mars 2001, accepté le 28 septembre 2001

Daniel Azagra, Departamento de Análisis Matemático, Facultad de Matemáticas, Universidad Complutense de Madrid, 28040 Madrid (Spain)

E-mail : daniel_azagra@mat.ucm.es • Url : http://www.mat.ucm.es/deptos/am/

Mar Jiménez-Sevilla, Departamento de Análisis Matemático, Facultad de Matemáticas,

Universidad Complutense de Madrid, 28040 Madrid (Spain)

E-mail : MM_Jimenez@Mat.UCM.Es •Url : http://ochoa.mat.ucm.es/ marjim/

2000 Mathematics Subject Classification. — 46B20, 58B99.

Key words and phrases. - Gradient, bump function, starlike body.

Supported in part by DGICYT grant PB 96-0607 and BFM 2000-0609. 
RÉsumé (Sur la taille des ensembles de dérivées des fonctions bosses et des hyperplans tangents aux corps étoilés dans l'espace de Hilbert)

On étudie la taille des ensembles de dérivées des fonctions bosses sur l'espace de Hilbert $\ell_{2}$, ainsi que celle de l'ensemble des hyperplans tangents à un corps étoilé dans $\ell_{2}$. On trouve que ces ensembles peuvent être assez petits. D'un côté, la norme de l'espace de Hilbert peut s'approximer uniformément par des fonctions de classe $C^{1}$ et lipschitziennes $\psi$ telles que les cônes générés par les images des dérivées $\psi^{\prime}\left(\ell_{2}\right)$ sont d'intérieur vide. Cela entraîne l'existence de fonctions de classe $C^{1}$ et lipschitziennes dont les cônes générés par les images des dérivées sont d'intérieur vide. On construit d'autre part des corps étoilés bornés lisses de classe $C^{1}$ et lipschitziens dont les cônes générés par leurs hyperplans tangents sont d'intérieur vide. On montre aussi pourquoi ces résultats constituent la meilleure réponse à ces questions que l'on puisse espérer.

\section{Introduction}

Smooth bump functions and starlike bodies are objects that arise naturally in non-linear functional analysis, and therefore their geometrical properties are worth studying. However, very natural questions about tangent hyperplanes to such objects have remained unasked or unanswered, even in the Hilbert space, until very recently.

For instance, if $b: X \rightarrow \mathbb{R}$ is a smooth bump on a Banach space $X$ (that is, a smooth function with a bounded support, not identically zero), how many tangent hyperplanes does its graph have? In other words, if we denote the cone generated by its set of gradients by

$$
\mathcal{C}(b)=\left\{\lambda b^{\prime}(x): x \in X, \lambda \geq 0\right\},
$$

what is the (topological) size of $\mathcal{C}(b)$ ?

This problem is strongly related to a similar question about the size of the cones of tangent hyperplanes to starlike bodies in $X$. Namely, if $A$ is a smooth bounded starlike body in $X$, how many tangent hyperplanes does $A$ have? More precisely, if we denote the cone of hyperplanes which are tangent to $A$ at some point of its boundary $\partial A$ by

$$
\mathcal{C}(A)=\left\{x^{*} \in X: x+\operatorname{Ker} x^{*} \text { is tangent to } \partial A \text { at some point } x \in \partial A\right\},
$$

what is the size of $\mathcal{C}(A)$ ?

Although in this paper we are mainly concerned with the case of the Hilbert space $\ell_{2}$, it may be helpful to make some previous general considerations about these questions.

To begin with, as a consequence of Ekeland's variational principle [4], it is easily seen that if $b: X \rightarrow \mathbb{R}$ is a Gâteaux smooth and continuous bump function on a Banach space $X$ then the norm-closure of $b^{\prime}(X)$ is a neighbourhood of 0 in $X^{*}$. If, in addition, $X$ is finite-dimensional, and $b$ is $C^{1}$ smooth,

TOME $130-2002-\mathrm{N}^{\mathrm{O}} 3$ 
then $b^{\prime}(X)$ is a compact neighbourhood of 0 in $X^{*}$, and in particular 0 is an interior point of $b^{\prime}(X)$.

However, the classical Rolle's theorem is false in a Banach space $X$ whenever there are smooth bumps in $X$ (see [2] and the references included therein), and this fact has some interesting consequences on the question about the minimal size of the cones of gradients $\mathcal{C}(b)$. Indeed, by using the main result of [2], one can construct smooth bump functions whose sets of gradients lack not only the point zero, but any pre-set finite-dimensional linear subspace of the dual space, so that they violate Rolle's theorem in a quite strong way, as we will see in Section 2.

If we restrict the scope of our search to classic Banach spaces, much stronger results are available. On the one hand, if $X=c_{0}$ the size of $\mathcal{C}(b)$ can be really small. Indeed, as a consequence of P. Hájek's work [6] on smooth functions on $c_{0}$ we know that if $b$ is $C^{1}$ smooth with a locally uniformly continuous derivative (note that there are bump functions with this property in $c_{0}$ ), then $b^{\prime}(X)$ is contained in a countable union of compact sets in $X^{*}$ (and in particular $\mathcal{C}(b)$ has empty interior). On the other hand, if $X$ is non-reflexive and has a Fréchet norm, there are Fréchet smooth bumps $b$ on $X$ so that $\mathcal{C}(b)$ has empty interior, as it was shown in [1].

In the reflexive case, however, the problem is far from being settled. To begin with, the cone $\mathcal{C}(b)$ cannot be very small, since it is going to be a residual subset of the dual $X^{*}$. Indeed, as a consequence of Stegall's variational principle (see [9]), for every Banach space $X$ having the Radon-Nikodym Property (RNP) it is not difficult to see that $\mathcal{C}(b)$ is a residual set in $X^{*}$. Thus, for infinitedimensional Banach spaces $X$ enjoying RNP (such is the case of reflexive ones and, of course, $\ell_{2}$ ) one can hardly expect a better answer to the question about the minimal size of the cones of gradients of smooth bumps than the following one: there are smooth bumps $b$ on $X$ such that the cones $\mathcal{C}(b)$ have empty interior in $X^{*}$.

In the same way, if $A$ is a bounded starlike body in a RNP Banach space then the cone $\mathcal{C}(A)$ of tangent hyperplanes to $A$ contains a subset of second Baire category in $X^{*}$, so the best result one could get about the smallest possible size of the cone of tangent hyperplanes to a starlike body in $\ell_{2}$ is that there exist smooth bounded starlike bodies $A$ in $\ell_{2}$ so that $\mathcal{C}(A)$ have empty interior.

In [1] a study was initiated on the topological size of the set of gradients of smooth functions and starlike bodies. Among other results it was proved that an infinite-dimensional Banach space has a $C^{1}$ smooth Lipschitz bump function if and only if there exists another $C^{1}$ smooth Lipschitz bump function $b$ on $X$ with the property that $b^{\prime}(X)$ contains the unit ball of the dual $X^{*}$ and, in particular, $\mathcal{C}(b)=X^{*}$. It was also established that James' theorem fails for starlike bodies, in the following senses. First, for every Banach space $X$ with a separable dual $X^{*}$, there exists a $C^{1}$ smooth Lipschitz and bounded starlike 
body $A_{1}$ so that $\mathcal{C}\left(A_{1}\right)=X^{*}$; in particular we see that there is no upper bound on the size of the cone $\mathcal{C}(A)$, even though $X$ is nonreflexive, and therefore the difficult part of James' theorem is false for starlike bodies. Second, there exists a $C^{1}$ smooth Lipschitz and bounded starlike body $A_{2}$ in $\ell_{2}$ so that $\mathcal{C}\left(A_{2}\right) \neq \ell_{2}$, and in particular the "easy" part of James' theorem is false too for starlike bodies.

While the first of these results fully answers the question about the maximal size of the cone $\mathcal{C}(A)$, the second one is not so conclusive, and the natural question as to how small $\mathcal{C}(A)$ can be remained open.

Here, in the case of the Hilbert space $X=\ell_{2}$, we provide full answers to the questions on the smallest possible size of the cones $\mathcal{C}(A)$ and $\mathcal{C}(b)$, for a smooth bounded starlike body $A$ in $X$ and a smooth bump function $b$ on $X$. In Sections 2 and 3 we construct $C^{1}$ smooth bumps $b$ and $C^{1}$ smooth starlike bodies $A$ in $\ell_{2}$ so that the cones of gradients $\mathcal{C}(b)$ and $\mathcal{C}(A)$ have empty interior. Moreover, these strange objects can be made to uniformly approximate the norm and the unit ball of $\ell_{2}$ respectively.

\section{How small can the set of gradients of a bump be?}

As said above, the question as to how small the cone of gradients of a bump can be is tightly related to the failure of Rolle's theorem in infinite-dimensional Banach spaces. We begin by showing how one can use the main result of [2] to construct smooth bump functions whose sets of gradients lack not only the point zero, but any pre-set finite-dimensional linear subspace of the dual space, so that they violate Rolle's theorem in a quite strong manner.

THEOREM 2.1. - Let $X$ be an infinite-dimensional Banach space and $W$ a finite-dimensional subspace of $X^{*}$. The following statements are equivalent.

1) $X$ has a $C^{p}$ smooth (Lipschitz) bump function.

2) $X$ has a $C^{p}$ smooth (Lipschitz) bump function $f$ so that $\mathcal{C}(f) \cap W=\{0\}$ and, moreover,

$$
\left\{f^{\prime}(x): x \in \operatorname{int}(\operatorname{supp}(f))\right\} \cap W=\varnothing .
$$

Proof. — We only need to prove that 1) implies 2). We can write $X=Y \oplus Z$, where $Y=\bigcap_{w^{*} \in W} \operatorname{ker} w^{*}$ and $\operatorname{dim} Z=\operatorname{dim} W$ is finite. Let us pick a $C^{p}$ smooth (Lipschitz) bump function $\varphi: Y \rightarrow \mathbb{R}$ such that $\varphi^{\prime}(y)=0$ if and only if $y \notin \operatorname{int}(\operatorname{supp}(\varphi))$ (the existence of such a bump $\varphi$ is guaranteed by Theorem 1.1 in [2]). Let $\theta$ be a $C^{\infty}$ smooth Lipschitz bump function on $Z$ so that $\theta^{\prime}(z)=0$ whenever $\theta(z)=0$. Then the function $f: X=Y \oplus Z \rightarrow \mathbb{R}$ defined by $f(y, z)=\varphi(y) \theta(z)$ is a $C^{p}$ smooth (Lipschitz) bump which satisfies $\left\{f^{\prime}(x): x \in \operatorname{int}(\operatorname{supp}(f))\right\} \cap W=\varnothing$. Indeed, if $(y, z) \in Y \oplus Z$ we have

$$
f^{\prime}(y, z)=\left(\theta(z) \varphi^{\prime}(y), \varphi(y) \theta^{\prime}(z)\right) \in X^{*}=Y^{*} \oplus Z^{*}=Y^{*} \oplus W .
$$

TOME $130-2002-\mathrm{N}^{\mathrm{O}} 3$ 
If $(y, z) \in \operatorname{int}(\operatorname{supp}(f))$, then $\theta(z) \varphi^{\prime}(y) \neq 0$, and hence $f^{\prime}(y, z) \notin W$ and $C(f) \cap W=\{0\}$.

The following theorem and its corollary are the main results of this section. This theorem is also the keystone for the construction of a smooth bounded starlike body whose cone of tangent hyperplanes has empty interior (see the next section).

ThEOREM 2.2. - Let $\|\cdot\|$ denote the usual hilbertian norm of $\ell_{2}$. There are $C^{1}$ functions $f_{\varepsilon}: \ell_{2} \rightarrow(0, \infty), 0<\varepsilon<1$, which are Lipschitz on bounded sets and have Lipschitz derivatives, so that:

1) $\lim _{\varepsilon \rightarrow 0} f_{\varepsilon}(x)=\|x\|^{2}$ uniformly on $\ell_{2}$;

2) $\lim _{\varepsilon \rightarrow 0} f_{\varepsilon}^{\prime}(x)=2 x$ uniformly on $\ell_{2}$ (that is, the derivatives of the $f_{\varepsilon}$ uniformly approximate the derivative of the squared norm of $\left.\ell_{2}\right)$; and

3 ) the cones $\mathcal{C}\left(f_{\varepsilon}\right)$ generated by the sets of gradients of the $f_{\varepsilon}$ have empty interior, and $f_{\varepsilon}^{\prime}(x) \neq 0$ for all $x \in \ell_{2}, 0<\varepsilon<1$.

Moreover, the functions $\psi_{\varepsilon}=\left(f_{\varepsilon}\right)^{\frac{1}{2}}$ are $C^{1}$ smooth and Lipschitz, with Lipschitz derivatives. Note, in particular, that $\lim _{\varepsilon \rightarrow 0} \psi_{\varepsilon}=\|\cdot\|$ uniformly on $\ell_{2}$, the cones of gradients $\mathcal{C}\left(\psi_{\varepsilon}\right)$ have empty interior, and $\psi_{\varepsilon}^{\prime}(x) \neq 0$ for all $x \in \ell_{2}$. Besides, for every $r>0$, the derivatives $\psi_{\varepsilon}^{\prime}$ approximate the derivative of the norm uniformly on the set $\left\{x \in \ell_{2}:\|x\| \geq r\right\}$ as $\varepsilon$ goes to 0 .

Corollary 2.3. - There is a $C^{1}$ Lipschitz bump function b on $\ell_{2}$ (with Lipschitz derivative) satisfying that the cone $\mathcal{C}(b)$ generated by its set of gradients has empty interior, and $b^{\prime}(x) \neq 0$ for every $x$ in the interior of its support.

\section{Proofs of Theorem 2.2 and Corollary 2.3}

We will make use of the following restatement of a striking result due to S.A. Shkarin (see [10]).

TheOREM 2.4 (Shkarin). - There is a $C^{\infty}$ diffeomorphism $\varphi$ from $\ell_{2}$ onto $\ell_{2} \backslash\{0\}$ such that all the derivatives $\varphi^{(n)}$ are uniformly continuous on $\ell_{2}$, and $\varphi(x)=x$ for $\|x\| \geq 1$.

Let us consider, for $0<\varepsilon<1$, the diffeomorphism $\varphi_{\varepsilon}: \ell_{2} \rightarrow \ell_{2} \backslash\{0\}, \varphi_{\varepsilon}(x)=$ $\varepsilon \varphi(x / \varepsilon)$, and the function $U \equiv U_{\varepsilon}: \ell_{2} \rightarrow \mathbb{R}$ defined by $U(x)=\varepsilon^{2}+\left\|\varphi_{\varepsilon}(x)\right\|^{2}$. Then $U$ satisfies the following properties:

(i) $U$ is $C^{\infty}$ smooth;

(ii) $\|x\|^{2} \leq U(x) \leq 2 \varepsilon^{2}+\|x\|^{2}$ and $\varepsilon^{2} \leq U(x)$, for every $x \in \ell_{2}$;

(iii) $U(x)=\varepsilon^{2}+\|x\|^{2}$, for every $x \in \ell_{2},\|x\| \geq \varepsilon$;

(iv) $U^{\prime}(x) \neq 0$ for every $x \in \ell_{2}$;

(v) $U$ is Lipschitz in bounded sets and $U^{\prime}$ is Lipschitz.

BULletin DE LA SOCIÉtÉ MATHÉMATIQUE DE FRANCE 
Now, we define the functions $U_{n}: \ell_{2} \rightarrow \mathbb{R}$ by

$$
U_{n}(x)=\frac{1}{2^{2 n}} U\left(2^{n} x\right),
$$

whenever $x \in \ell^{2}$. We identify $\ell_{2}$ with the infinite sum $\sum_{2} \ell_{2} \equiv \ell_{2} \oplus_{2} \ell_{2} \oplus_{2} \ell_{2} \cdots$, where an element $x=\left(x_{n}\right)$ belongs to $\sum_{2} \ell_{2}$ if and only if every $x_{n}$ is in $\ell_{2}$ and $\sum_{n}\left\|x_{n}\right\|^{2}<\infty$, being $\|x\|^{2}=\sum_{n}\left\|x_{n}\right\|^{2}$. Then, we define the function $f \equiv f_{\varepsilon}: \sum_{2} \ell_{2} \rightarrow \mathbb{R}$ by

$$
f(x)=\sum_{n} U_{n}\left(x_{n}\right), \text { where } x=\left(x_{n}\right)_{n} .
$$

First, note that $f$ is well-defined, since condition (ii) implies that, whenever $x=\left(x_{n}\right) \in \sum_{2} \ell_{2}$,

$$
\begin{aligned}
0<f(x) & =\sum_{n} \frac{1}{2^{2 n}} U\left(2^{n} x_{n}\right) \\
& \leq \sum_{n} \frac{1}{2^{2 n}}\left(2 \varepsilon^{2}+\left\|2^{n} x_{n}\right\|^{2}\right)=\sum_{n}\left(\frac{2 \varepsilon^{2}}{2^{2 n}}+\left\|x_{n}\right\|^{2}\right)<\infty .
\end{aligned}
$$

On the other hand, if $U^{\prime}$ has Lipschitz constant $M$ then $U_{n}^{\prime}$ is also Lipschitz with constant $M$, since for $x$ and $y$ in $\ell_{2}$ we have

$$
\left\|U_{n}^{\prime}(x)-U_{n}^{\prime}(y)\right\|=\frac{1}{2^{n}}\left\|U^{\prime}\left(2^{n} x\right)-U^{\prime}\left(2^{n} y\right)\right\| \leq M\|x-y\| .
$$

This implies that, if $x=\left(x_{n}\right) \in \sum_{2} \ell_{2}$, the functionals $U_{n}^{\prime}\left(x_{n}\right) \in \ell_{2}$ satisfy that $\left(U_{n}^{\prime}\left(x_{n}\right)\right)_{n} \in\left(\sum_{2} \ell_{2}\right)^{*} \equiv \sum_{2} \ell_{2}$. Indeed, we have $\left\|U_{n}^{\prime}\left(x_{n}\right)-U_{n}^{\prime}(0)\right\| \leq M\left\|x_{n}\right\|$, and therefore $\sum_{n}\left\|U_{n}^{\prime}\left(x_{n}\right)-U_{n}^{\prime}(0)\right\|^{2}<\infty$. Also, $\left(U_{n}^{\prime}(0)\right)=\left(2^{-n} U^{\prime}(0)\right) \in$ $\sum_{2} \ell_{2}$, and then we obtain that $T(x) \equiv\left(U_{n}^{\prime}\left(x_{n}\right)\right)$ also belongs to $\sum_{2} \ell_{2}$.

Let us now prove that $f$ is $C^{1}$ smooth. For every $x=\left(x_{n}\right)$ and $h=\left(h_{n}\right)$ in $\sum_{2} \ell_{2}$, we can estimate

$$
\begin{aligned}
& |f(x+h)-f(x)-T(x)(h)| \leq \sum_{n}\left|U_{n}\left(x_{n}+h_{n}\right)-U_{n}\left(x_{n}\right)-U_{n}^{\prime}\left(x_{n}\right)\left(h_{n}\right)\right| \\
& \quad \leq \sum_{n}\left|U_{n}^{\prime}\left(x_{n}+t_{n} h_{n}\right)\left(h_{n}\right)-U_{n}^{\prime}\left(x_{n}\right)\left(h_{n}\right)\right| \quad\left(\text { for some } 0 \leq t_{n} \leq 1\right) \\
& \quad \leq M \sum_{n}\left\|h_{n}\right\|^{2}=M\|h\|^{2} .
\end{aligned}
$$

Therefore $f$ is Fréchet differentiable and $f^{\prime}(x)=T(x)$. Moreover, $f^{\prime}$ is Lipschitz since $\left\|f^{\prime}(x)-f^{\prime}(y)\right\|^{2}=\sum_{n}\left\|U_{n}^{\prime}\left(x_{n}\right)-U_{n}^{\prime}\left(y_{n}\right)\right\|^{2} \leq M^{2} \sum_{n}\left\|x_{n}-y_{n}\right\|^{2}=$ $M^{2}\|x-y\|^{2}$. This implies, in particular, that $f$ is Lipschitz on bounded sets.

Let us check that $f \equiv f_{\varepsilon}$ uniformly approximates $\|\cdot\|^{2}$ as $\varepsilon$ goes to 0 . Indeed, from condition (ii) on $U$ and inequality (1), we have that, for every $x=\left(x_{n}\right)$

TOME $130-2002-\mathrm{N}^{\mathrm{O}} 3$ 
in $\sum_{2} \ell_{2}$,

$$
\max \left\{\frac{1}{3} \varepsilon^{2},\|x\|^{2}\right\} \leq f(x) \leq \frac{2}{3} \varepsilon^{2}+\|x\|^{2}
$$

and then,

$$
0 \leq f(x)-\|x\|^{2} \leq \frac{2}{3} \varepsilon^{2} .
$$

In order to obtain functions which approximate the norm uniformly in $\ell_{2}$ let us consider $\psi \equiv \psi_{\varepsilon}=\sqrt{f_{\varepsilon}}$. According to inequalities (3) and (4) we have that

$$
0 \leq \psi-\|x\| \leq \frac{2 \varepsilon^{2}}{3(\psi+\|x\|)} \leq \frac{2}{\sqrt{3}} \varepsilon
$$

for any $x \in \sum_{2} \ell_{2}$.

Let us check that $\psi^{\prime}$ is bounded. By inequalities (2) and (4) we have, for any $x \in \sum_{2} \ell_{2}$,

$$
\left\|\psi^{\prime}(x)\right\|=\frac{\left\|f^{\prime}(x)\right\|}{2 \psi(x)} \leq \frac{\left\|f^{\prime}(x)-f^{\prime}(0)\right\|}{2 \psi(x)}+\frac{\left\|f^{\prime}(0)\right\|}{2 \psi(x)} \leq \frac{M}{2}+\frac{\sqrt{3}}{2 \varepsilon}\left\|f^{\prime}(0)\right\| .
$$

Consequently, $\psi$ is Lipschitz with Lipschitz constant, say $N$. In a similar way, we obtain that $\psi^{\prime}$ is Lipschitz, since for any $x, y$ in $\sum_{2} \ell_{2}$,

$$
\begin{aligned}
\left\|\psi^{\prime}(x)-\psi^{\prime}(y)\right\| & =\left\|\frac{f^{\prime}(x)-f^{\prime}(y)}{2 \psi(x)}+\frac{f^{\prime}(y)}{2}\left(\frac{1}{\psi(x)}-\frac{1}{\psi(y)}\right)\right\| \\
& \leq \frac{1}{2} \frac{\left\|f^{\prime}(x)-f^{\prime}(y)\right\|}{\psi(x)}+\frac{\|\psi(y)-\psi(x)\|}{\psi(x)} \cdot \frac{\left\|f^{\prime}(y)\right\|}{2 \psi(y)} \\
& \leq \frac{\sqrt{3} M}{2 \varepsilon}\|x-y\|+\frac{\sqrt{3} N^{2}}{\varepsilon}\|x-y\| .
\end{aligned}
$$

Let us now see that the derivatives of $f_{\varepsilon}$ uniformly approximates the derivative of $\|\cdot\|^{2}$ as $\varepsilon$ tends to 0 . Following the same notation as above, let us take $0<\delta<1$ and consider, for

$$
0<\varepsilon \leq \frac{\delta}{2+M+2\|\varphi(0)\| \cdot\left\|\varphi^{\prime}(0)\right\|}<\frac{\delta}{2}
$$

the associated mappings $f_{\varepsilon}(x) \equiv f(x)=\sum_{n} U_{n}\left(x_{n}\right)$, where $x=\left(x_{n}\right) \in \sum_{2} \ell_{2}$. It is straightforward to verify that

(a) $U_{n}\left(x_{n}\right)=2^{-2 n} \varepsilon^{2}+\left\|x_{n}\right\|^{2}$ for all $x_{n} \in \ell_{2}$ with $\left\|x_{n}\right\| \geq 2^{-n} \varepsilon$; and

(b) $U_{n}^{\prime}(0)=2^{-n} \cdot 2 \varepsilon \varphi(0) \varphi^{\prime}(0)$, for every $n \in \mathbb{N}$.

Given $x=\left(x_{n}\right) \in \sum_{2} \ell_{2}$, we define $D=\left\{n \in \mathbb{N}:\left\|x_{n}\right\| \leq 2^{-n} \varepsilon\right\}$. From the above properties (a) and (b), and the Lipschitz condition on $U_{n}^{\prime}$ provided by BULletin DE LA SOCiÉtÉ MATHÉmATiQUe DE FRANCE 
equation (2), we deduce the following inequalities

$$
\begin{aligned}
& \left\|f^{\prime}(x)-2 x\right\|=\left(\sum_{n}\left\|U_{n}^{\prime}\left(x_{n}\right)-2 x_{n}\right\|^{2}\right)^{\frac{1}{2}}=\left(\sum_{D}\left\|U_{n}^{\prime}\left(x_{n}\right)-2 x_{n}\right\|^{2}\right)^{\frac{1}{2}} \\
& \leq\left(\sum_{D}\left\|U_{n}^{\prime}\left(x_{n}\right)-U_{n}^{\prime}(0)\right\|^{2}\right)^{\frac{1}{2}}+\left(\sum_{D}\left\|U_{n}^{\prime}(0)\right\|^{2}\right)^{\frac{1}{2}}+\left(\sum_{D}\left\|2 x_{n}\right\|^{2}\right)^{\frac{1}{2}} \\
& \leq M\left(\sum_{D}\left\|x_{n}\right\|^{2}\right)^{\frac{1}{2}}+2 \varepsilon\|\varphi(0)\| \cdot\left\|\varphi^{\prime}(0)\right\|+2\left(\sum_{D}\left\|x_{n}\right\|^{2}\right)^{\frac{1}{2}},
\end{aligned}
$$

and therefore

$$
\left\|f_{\varepsilon}^{\prime}(x)-2 x\right\| \leq \varepsilon\left(M+2+2\|\varphi(0)\| \cdot\left\|\varphi^{\prime}(0)\right\|\right) \leq \delta .
$$

This shows that $\lim _{\varepsilon \rightarrow 0} f_{\varepsilon}^{\prime}(x)=2 x$ uniformly on $\ell_{2}$.

Finally, let us see that the cones of gradients $\mathcal{C}\left(f_{\varepsilon}\right)$ have empty interior. It suffices to note that the set $\left\{\lambda f^{\prime}(x)=\lambda\left(U_{n}^{\prime}\left(x_{n}\right)\right): x=\left(x_{n}\right) \in \sum_{2} \ell_{2}, \lambda>0\right\}$ is contained in $\left\{z=\left(z_{n}\right) \in \sum_{2} \ell_{2}: z_{n} \neq 0\right.$ for every $\left.n \in \mathbb{N}\right\}$, which has empty interior in $\sum_{2} \ell_{2}$. This concludes the proof of Theorem 2.2.

In order to prove Corollary 2.3, we consider a $C^{\infty}$ function $\theta: \mathbb{R}^{+} \rightarrow \mathbb{R}$, $\theta^{\prime}(t)<0$ for $t \in(0,1)$, and $\operatorname{supp} \theta=(0,1]$. Then, we can define a required bump function as the composition $b(x)=\theta(f(x))$. Indeed, on the one hand, $0<f(0) \leq \frac{2}{3} \varepsilon^{2}<1$ and therefore $b(0)>0$. On the other hand, $f(x) \geq\|x\|^{2} \geq 1$, whenever $\|x\| \geq 1$, and hence $b(x)=0$ for $\|x\| \geq 1$. The bump function $b$ is clearly Lipschitz with Lipschitz derivative since $\theta, \theta^{\prime}$ and $f^{\prime}$ are Lipschitz and $f$ is Lipschitz on bounded sets.

\section{Geometrical properties of starlike bodies in $\ell_{2}$}

A closed subset $A$ of a Banach space $X$ is said to be a starlike body provided $A$ has a non-empty interior and there exists a point $x_{0} \in \operatorname{int} A$ such that each ray emanating from $x_{0}$ meets the boundary of $A$ at most once. In this case we will say that $A$ is starlike with respect to $x_{0}$. When dealing with starlike bodies, we can always assume that they are starlike with respect to the origin (up to a suitable translation). For a starlike body $A$, we define the Minkowski functional of $A$ as

$$
\mu_{A}(x)=\inf \left\{\lambda>0: \frac{1}{\lambda} x \in A\right\}
$$

for all $x \in X$. It is easily seen that $\mu_{A}$ is a continuous function which satisfies $\mu_{A}(r x)=r \mu_{A}(x)$ for every $r \geq 0$. Moreover, $A=\left\{x \in X: \mu_{A}(x) \leq 1\right\}$, and $\partial A=\left\{x \in X: \mu_{A}(x)=1\right\}$, where $\partial A$ stands for the boundary of $A$. Conversely, if $\psi: X \rightarrow[0, \infty)$ is continuous and satisfies $\psi(\lambda x)=\lambda \psi(x)$ for all $\lambda \geq 0$, then $A_{\psi}=\{x \in X: \psi(x) \leq 1\}$ is a starlike body. Convex bodies are

TOME $130-2002-\mathrm{N}^{\mathrm{O}} 3$ 
an important kind of starlike bodies. We will say that $A$ is a $C^{p}$ smooth (Lipschitz) starlike body provided its Minkowski functional $\mu_{A}$ is $C^{p}$ smooth (and Lipschitz) on the set $X \backslash \mu_{A}^{-1}(0)$.

It is worth noting that every Banach space having a $C^{p}$ smooth (Lipschitz) bump function has a $C^{p}$ smooth (Lipschitz) bounded starlike body too [4] (and the converse is also true). Level sets of $n$-homogeneous polynomials in Banach spaces are always boundaries of smooth starlike bodies. This is not true, in general, of level sets of smooth bump functions.

Recall that we denote the cone of hyperplanes which are tangent to $A$ at some point of its boundary $\partial A$ by

$$
\mathcal{C}(A)=\left\{x^{*} \in \ell_{2}: x+\operatorname{Ker} x^{*} \text { is tangent to } \partial A \text { at some point } x \in \partial A\right\} .
$$

Note also that

$$
\mathcal{C}(A)=\mathcal{C}\left(\mu_{A}\right):=\left\{\lambda \mu_{A}^{\prime}(x): x \in X, x \neq 0, \lambda \geq 0\right\} .
$$

The next result fully answers the question as to how small $\mathcal{C}(A)$ can be in the Hilbert space.

THEOREM 3.1. - There are $C^{1}$ smooth Lipschitz and bounded starlike bodies $A_{\varepsilon}$ in $\ell_{2}, 0<\varepsilon<1$, so that:

(i) their Minkowski functionals $\mu_{A_{\varepsilon}}$ uniformly approximate the usual norm on bounded sets, that is, $\lim _{\varepsilon \rightarrow 0} \mu_{A_{\varepsilon}}=\|\cdot\|$ uniformly on bounded sets of $\ell_{2}$; and

(ii) the cones $\mathcal{C}\left(A_{\varepsilon}\right)$ generated by the set of gradients of $\mu_{A_{\varepsilon}}$ have empty interior in $\ell_{2}$.

Proof. - We use the same notation as in the proof of Theorem 2.2. Take $0<\delta<1$ and consider, for $0<\varepsilon \leq \delta /\left(2+M+2\|\varphi(0)\| \cdot\left\|\varphi^{\prime}(0)\right\|\right)<\frac{1}{2} \delta$, the associated mapping $f_{\varepsilon}(x) \equiv f(x)=\sum_{n} U_{n}\left(x_{n}\right)$, where $x=\left(x_{n}\right) \in \sum_{2} \ell_{2}$. Now define $A_{\varepsilon}$ as

$$
A_{\varepsilon} \equiv A=\left\{x \in \ell_{2}: f(x) \leq 1\right\} .
$$

Clearly $A$ is a closed set with boundary

$$
\partial A=\left\{x \in \ell_{2}: f(x)=1\right\},
$$

and by inequality (4) we have the inclusion

$$
(1-\varepsilon) B \subset A \subset B,
$$

where $B$ denotes the unit ball of $\ell_{2}$. In particular, this proves (i).

In order to show that $A$ is a starlike body (with respect to 0 ) we must bear in mind the fact that the $f_{\varepsilon}^{\prime}$ approximate the derivative of $\|\cdot\|^{2}$; in particular we shall use inequality (5). Suppose that there is a norm-one element $x \in \sum_{2} \ell_{2}$ and $\lambda, \nu>0, \lambda \neq \nu$ satisfying $f(\lambda x)=f(\nu x)=1$, then there is $\tau \in(\lambda, \nu)$ so that $0=f(\lambda x)-f(\nu x)=f^{\prime}(\tau x)(x)(\lambda-\nu)$. Thus, taking into account inequality (5) and the fact that $\tau>1-\varepsilon$, we have that

$$
0=f^{\prime}(\tau x)(x)=\langle 2 \tau x, x\rangle+\left\langle f^{\prime}(\tau x)-2 \tau x, x\right\rangle \geq 2 \tau-\delta>2-2 \delta>0,
$$

BULLETIN DE LA SOCIÉtÉ MATHÉMATIQUE DE FRANCE 
which is a contradiction.

Let us now show that the Minkowski functional $\mu_{A}$ is $C^{1}$ smooth. Consider the $C^{1}$ smooth function

$$
F:\left(\sum_{2} \ell_{2} \backslash \frac{1}{2} B_{1}\right) \times\left(\frac{1}{2}, \infty\right) \longrightarrow \mathbb{R}, \quad F(x, t)=f\left(\frac{x}{t}\right) .
$$

The functional $\mu_{A}$ satisfies the implicit equation $F\left(x, \mu_{A}(x)\right)=1$. Also,

$$
\begin{aligned}
\frac{\partial F}{\partial t}\left(x, \mu_{A}(x)\right) & =-\frac{1}{\mu_{A}^{2}(x)}\left\langle f^{\prime}\left(x / \mu_{A}(x)\right), x\right\rangle \\
& =-\frac{1}{\mu_{A}^{2}(x)}\left(\left\langle\frac{2 x}{\mu_{A}(x)}, x\right\rangle+\left\langle f^{\prime}\left(\frac{x}{\mu_{A}(x)}\right)-\frac{2 x}{\mu_{A}(x)}, x\right\rangle\right) \\
& \leq-\frac{\|x\|}{\mu_{A}^{2}(x)}\left(\frac{2\|x\|}{\mu_{A}(x)}-\delta\right) .
\end{aligned}
$$

Since $1-\frac{1}{2} \delta<1-\varepsilon \leq\left\|x / \mu_{A}(x)\right\| \leq 1$ we conclude that

$$
\frac{\partial F}{\partial t}\left(x, \mu_{A}(x)\right) \leq-\frac{\|x\|}{\mu_{A}^{2}(x)}(2-2 \delta)<0 .
$$

Thus, by the implicit function theorem, it follows that the mapping $\mu_{A}$ is $C^{1}$ smooth.

Let us next prove that $\mu_{A}^{\prime}$ is bounded in $\sum_{2} \ell_{2} \backslash\{0\}$, and therefore $\mu_{A}$ is Lipschitz. We derive the implicit equation $f\left(x / \mu_{A}(x)\right)=1$ and obtain

$$
0=\frac{1}{\mu_{A}(x)} f^{\prime}\left(\frac{x}{\mu_{A}(x)}\right)-\frac{1}{\mu_{A}(x)^{2}}\left\langle f^{\prime}\left(\frac{x}{\mu_{A}(x)}\right), x\right\rangle \mu_{A}^{\prime}(x) .
$$

Since $\mu_{A}^{\prime}(\lambda x)=\mu_{A}^{\prime}(x)$, whenever $\lambda>0$, it is sufficient to show that $\mu_{A}^{\prime}$ is bounded on the set $\left\{x \in \sum_{2} \ell_{2}: \mu_{A}(x)=1\right\}$. From equation (7) we obtain that

$$
\mu_{A}^{\prime}(x)=\frac{f^{\prime}(x)}{\left\langle f^{\prime}(x), x\right\rangle} \quad \text { whenever } \mu_{A}(x)=1 .
$$

From inequality (6) it follows that $\left\langle f^{\prime}(x), x\right\rangle \geq\|x\|(2-2 \delta) \geq(1-\varepsilon)(2-2 \delta)$ for $\mu_{A}(x)=1$, and this implies $\left\|\mu_{A}^{\prime}(x)\right\| \leq(1-\varepsilon)^{-1}(2-2 \delta)^{-1}\left\|f^{\prime}(x)\right\|$, which proves the assertion.

Finally, let us note that equation (7) implies the inclusion of the cones $\mathcal{C}\left(\mu_{A}\right) \subseteq \mathcal{C}(f)$ and, since $\mathcal{C}(f)$ has empty interior, so does $\mathcal{C}\left(\mu_{A}\right)$.

Acknowledgements. - This research was carried out during a stay of the authors in the Équipe d'Analyse de l'Université Pierre et Marie Curie, Paris 6. The authors are indebted to the Équipe d'Analyse and very especially to Gilles Godefroy for their kind hospitality and generous advice.

TOME $130-2002-\mathrm{N}^{\mathrm{O}} 3$ 


\section{BIBLIOGRAPHY}

[1] Azagra (D.) \& Deville (R.) - James' theorem fails for starlike bodies, J. Funct. Anal., t. 180 (2001), pp. 328-346.

[2] Azagra (D.) \& Jiménez-Sevilla (M.) - The failure of Rolle's theorem in infinite dimensional Banach spaces, J. Funct. Anal., t. 182 (2001), pp. 207-226.

[3] Borwein (J.M.), Fabian (M.), Kortezov (I.) \& Loewen (P.D.) The range of the gradient of a continuously differentiable bump, J. Nonlinear Convex Anal., t. 2 (2001), no. 1, pp. 1-19.

[4] Deville (R.), Godefroy (G.) \& Zizler (V.) - Smoothness and renormings in Banach spaces, Monographies and Surveys in Pure and Applied Math., vol. 64, Pitman, 1993.

[5] Ferrer (J.) - Rolle's theorem for polynomials of degree four in a Hilbert space, J. Math. Anal. Appl., t. 265 (2002), no. 2, pp. 322-331.

[6] HÁJeK (P.) - Smooth functions on $c_{0}$, Israel J. Math., t. 104 (1998), pp. 17-27.

[7] Jiménez-Sevilla (M.) \& Moreno (J.P.) - A note on norm attaining functionals, Proc. Amer. Math. Soc., t. 126 (1998), no. 3, pp. 1989-1997.

[8] MaLÝ (J.) - The Darboux property for gradients, Real Anal. Exchange, t. $22(1996 / 1997)$, pp. 167-173.

[9] Phelps (R.) - Convex functions, monotone operators and differentiability, Lecture Notes in Math., vol. 1364, Springer-Verlag, Berlin, 1989.

[10] Shkarin (S.A.) - On Rolle's theorem in infinite-dimensional Banach spaces, Mat. Zametki, t. 51 (1992), no. 3, pp. 128-136, English transl. 